BMJ Open

Diabetes

Research

\& Care

\title{
Hispanic representation in diabetes cardiovascular outcomes trials
}

\author{
A. Taylor Kelley, ${ }^{\oplus 1,2,3,4}$ Kara Mizokami-Stout,, ${ }^{1,2,3,4,5}$ Matthew J. O'Brien, ${ }^{6}$ \\ Michael E. Bowen, ${ }^{7}$ Jeremy Sussman ${ }^{1,2,3}$
}

To cite: Kelley AT, Mizokami-Stout K, 0'Brien MJ, et al. Hispanic representation in diabetes cardiovascular outcomes trials. BMJ Open Diab Res Care 2019;7:e000656. doi:10.1136/ bmjdrc-2019-000656

- Additional material is published online only. To view please visit the journal online (http://dx.doi.org/10.1136/ bmjdrc-2019-000656)

Received 16 January 2019 Revised 22 March 2019 Accepted 12 April 2019
Check for updates

\section{(c) Author(s) (or their} employer(s)) 2019. Re-use permitted under CC BY-NC. No commercial re-use. See rights and permissions. Published by BMJ.

For numbered affiliations see end of article.

Correspondence to Dr A. Taylor Kelley, Internal Medicine, University of Michigan, Ann Arbor, Michigan, USA; atkelley@med.umich.edu

\section{ABSTRACT}

Objective To examine Hispanic/Latino representation in diabetes cardiovascular outcomes trials for novel antidiabetic drugs.

Research design and methods We compared Hispanic/ Latino representation, age, gender and body mass index in diabetes cardiovascular outcomes trials published from January 2008 to October 2018 to Hispanic adults with diabetes in the National Health Examination and Nutrition Survey over the same time period.

Results Hispanics/Latinos comprised $18.5 \%$ of trial subjects, which was similar to the proportion of US adults with diabetes who identify as Hispanic. Trial subjects were significantly younger, more likely to be female, and more obese than US Hispanics/Latinos. At least 10 different Latin American countries and territories were represented across the 10 trials.

Conclusions US Hispanics/Latinos differ from subjects in diabetes cardiovascular outcomes trials, which may limit generalizability of trial results.

\section{INTRODUCTION}

The US Food and Drug Administration (FDA) is currently re-evaluating the role of diabetes cardiovascular outcomes trials (CVOTs) in determining the safety of new drugs for type 2 diabetes. ${ }^{1}$ An important consideration will be the degree to which results from these trials can be generalized to patients with type 2 diabetes, both domestically and internationally.

Type 2 diabetes in the USA disproportionately affects adults from all racial and ethnic minority groups, including Hispanics/ Latinos. ${ }^{2}$ For example, the prevalence of diabetes in Hispanics/Latinos is twice that of non-Hispanic whites, Hispanics/Latinos with diabetes are three times as likely as non-Hispanic whites to develop end stage renal disease, and diabetes-related mortality is $50 \%$ higher. ${ }^{34}$

Additionally, disparities in treatment response to novel diabetes therapeutics, such as oral and injectable antidiabetic drugs, have been observed in clinical trials across racial and ethnic minority groups, including Hispanics/Latinos. ${ }^{5}$ Recent studies have identified certain genetic markers

\section{Significance of this study}

What is already known about this subject?

- Incident type 2 diabetes is disproportionately high among US Hispanics/Latinos.

What are the new findings?

- Hispanics/Latinos included in diabetes cardiovascular outcomes trials differ from US Hispanics/Latinos in terms of age, gender, body mass index and country of origin, which may limit generalizability of trial results.

How might these results change the focus of research or clinical practice?

- The utility of diabetes cardiovascular outcomes trials may be enhanced by consistent reporting of Hispanic/Latino ethnicity and country of origin.

- Hispanic/Latino representation in diabetes cardiovascular outcomes trials may be improved to better reflect the US population. Clinicians should use caution in generalizing trial findings to their patients.

disproportionately prevalent in some US Hispanic/Latino populations-especially those with origins from Mexico-that may partially explain observed disparities in diabetes prevalence and outcomes and may provide a basis for heterogeneity of treatment effects. ${ }^{6}$ However, the number of clinical trials reporting outcomes for Hispanics/Latinos has historically been low. ${ }^{7}$

To address these disparities, the FDA has encouraged enrollment of racial/ethnic minorities in clinical trials and has provided guidance about how race and ethnicity should be reported in trial findings. ${ }^{8}$ However, Hispanic/Latino representation for CVOTs has not been summarized across studies. Our objective was to examine the representation of Hispanic/Latino ethnicity in CVOTs compared with the entire population of US Hispanic/Latino adults with diabetes over the same time period to determine how international trial results may apply to Hispanics/ Latinos in the USA. 


\section{Methods}

We reviewed 10 of 13 CVOTs with primary study results published January 2008 to October 2018. ' Trials were excluded if retrospective data were used $(n=1)$, research subjects in the USA were not included $(n=1)$, or the intervention was not an oral or injectable antidiabetic drug $(n=1) \cdot{ }^{9-19}$ A comprehensive search strategy is available in the online supplementary file 1 .

We next extracted and compiled available data on race, ethnicity, country of origin, age, gender, and body mass index (BMI) found in manuscript bodies or associated tables and appendices. All trial data were weighted according to trial sample size by dividing the sum of subjects with a given characteristic in each trial by the sum of all subjects across all trials. We extracted data for the same variables from the National Health and Nutrition Examination Survey (NHANES) from years 20072008 to 2015-2016, approximating the time interval from initiation to completion of CVOTs. ${ }^{20}$ For NHANES, we used classifications of Hispanic (self-report of Hispanic/ Latino ethnicity) and Mexican-American (a subset of Hispanic/Latino ethnicity that applies if the respondent reports that he or she or his or her ancestors come from Mexico) as comparison groups for CVOT reporting of Hispanic/Latino ethnicity. Finally, we compared all selected demographic variables between CVOTs and NHANES by ethnicity.
Results

All 10 trials included categories for race, but Hispanic/ Latino ethnicity was reported in only 8 of 10 trials. The proportion of Mexican-Americans was not reported in any trial.

Of the eight trials reporting Hispanic/Latino ethnicity, representation ranged from $12.1 \%$ to $29.1 \%$, with a composite weighted mean of $18.5 \%$. Among adults self-reporting diabetes in NHANES, respondents identifying as Hispanic/Latino increased from $11.9 \%$ in $2007-$ 2008 to $18.2 \%$ in 2015-2016, and respondents identifying as Mexican-American increased from $7.6 \%$ to $11.8 \%$. Compared with adult Hispanics/Latinos who report having diabetes in NHANES during 2015-2016, CVOT subjects on average were older (age 63.6 years vs 54.4 years), less likely to be women $(33.2 \%$ vs $48.2 \%)$, and less obese (BMI 31.0 vs 32.9). Trends for Mexican-Americans were similar to all Hispanics/Latinos in the NHANES data (see table 1).

Of the 10 trials, subjects were enrolled from at least 10 different Latin American countries or territories, including Argentina, ${ }^{9}$ Mexico, ${ }^{9}$ Brazil,${ }^{8}$ Chile,${ }^{5}$ Colombia, ${ }^{5}$ Peru, ${ }^{4}$ Guatemala, ${ }^{2}$ Ecuador, ${ }^{1}$ and Puerto Rico ${ }^{1}$ (see table 2). In terms of sample size, baseline characteristics, and outcomes, none of the reported data identified which or how many Hispanic/Latino subjects were from each Latin American country or territory and the USA.

Table 1 Ethnic composition, age, gender, and BMI among all adults and Hispanic/Latino subgroups with diabetes and NHANES and 10 CVOTs

\begin{tabular}{|c|c|c|c|c|}
\hline & $\begin{array}{l}\text { NHANES 2007-2008* } \\
(\%)\end{array}$ & $\begin{array}{l}\text { NHANES 2015-2016* } \\
(\%)\end{array}$ & $\begin{array}{l}\text { Weighted trial composite } \\
(\%)\end{array}$ & $\begin{array}{l}\text { Trial range } \\
(\%)\end{array}$ \\
\hline \multicolumn{5}{|c|}{ Racial/Ethnic Composition } \\
\hline Hispanic/Latino & 11.9 & 18.2 & 18.5 & $12.2-29.1$ \\
\hline Mexican American & 7.6 & 11.8 & - & \\
\hline \multicolumn{5}{|l|}{ Age (mean)§ } \\
\hline All & 59.3 & 59.9 & 63.6 & $60.3-65.2$ \\
\hline Hispanic/Latino & 56.9 & 54.4 & & \\
\hline Mexican American & 56.4 & 54.1 & & \\
\hline \multicolumn{5}{|l|}{ Female } \\
\hline All & 52.5 & 44.9 & 33.2 & 28.5-39.3 \\
\hline Hispanic/Latinoף & 54.1 & 48.2 & & \\
\hline Mexican-American & 52.0 & 46.9 & & \\
\hline \multicolumn{5}{|l|}{ BMI (mean) } \\
\hline All & 32.8 & 32.9 & 31.0 & $28.7-32.8$ \\
\hline Hispanic/Latino & 31.7 & 32.9 & & \\
\hline Mexican-American & 31.6 & 33.2 & & \\
\hline
\end{tabular}

*The definition for diabetes used in NHANES was self-report.

†Trial composition was weighted according to the size of the sample population for each trial.

$\ddagger$ Range was defined by the lowest and highest averages among the included trials for each variable.

$\S$ Some trials reported median rather than mean.

IData have been reported for one trial (TECOS).

BMI, body mass index; CVOTs, cardiovascular outcomes trials; NHANES, National Health and Nutrition Examination Survey. 


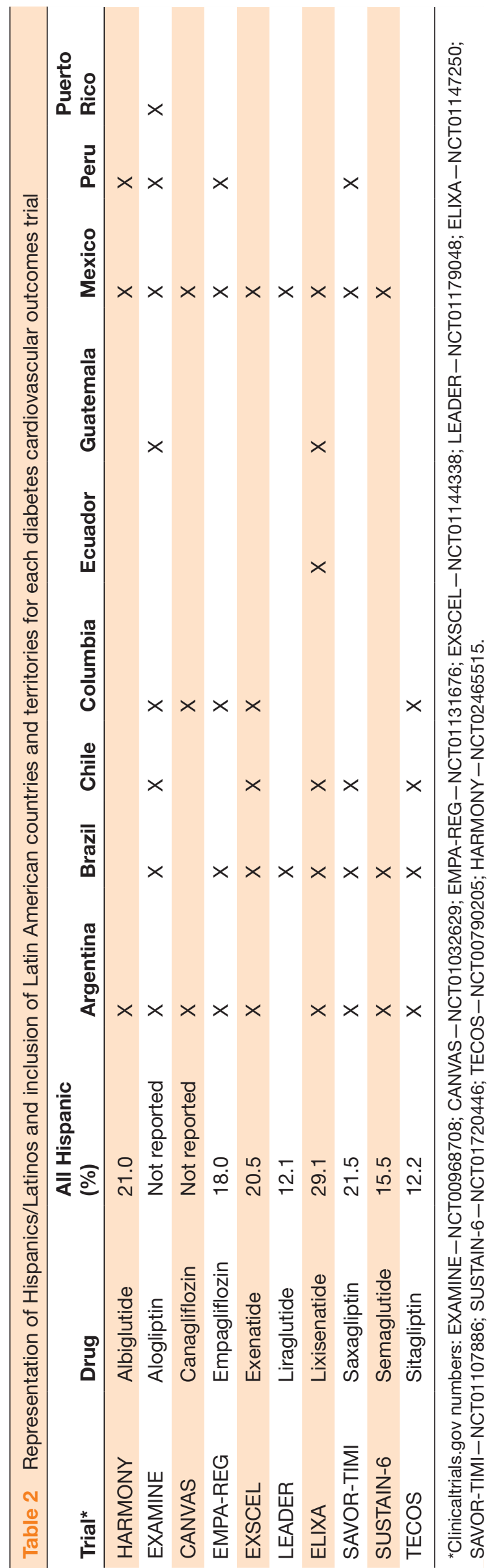

\section{Discussion}

The proportion of Hispanics/Latinos in CVOTs was similar to the population of US adults with diabetes as a whole. However, our findings raise questions about the generalizability of CVOT results among the US Hispanic/ Latino population. Hispanics/Latinos in CVOTs were older, were more likely to be male and had a lower BMI than population-based estimates.

Furthermore, in CVOT reports, it was not possible to determine the proportion of Hispanics/Latinos who were recruited in the USA compared with other countries. Nearly two-thirds of US Hispanics/Latinos with diabetes identify as Mexican-American, whereas Hispanics/Latinos in CVOTs were enrolled in at least eight countries and territories other than the USA or Mexico. This is a significant limitation to trial generalizability, given the large variation observed in diabetes prevalence across Latin American countries, as well as differences in diabetes prevalence among Hispanics/ Latinos in the USA compared with their countries of origin. ${ }^{21}$ Additionally, prior research on US Hispanics/ Latinos has documented an association of nativity and duration of US residence with diabetes. ${ }^{22}$ A possible interaction of genetic susceptibility and environmental factors may lead to higher incidence of diabetes for Hispanics/ Latinos when living in the USA than when living in their native countries. Whether cardiovascular safety of novel antidiabetic agents used among Hispanics living in their native countries can be generalized to Hispanics/Latinos living in the USA-where the risk of diabetes itself is increased-merits further study.

This study has several limitations. First, the nature of Hispanic/Latino identity and heritage is complex. NHANES survey questions do not distinguish Mexican-Americans born in Mexico from those born in the USA with Mexican ancestry. By contrast, many subjects in international CVOTs were often not US residents at all. It is possible that the impact of Hispanic/Latino ethnicity on the effects of diabetes treatment could be different between US and non-US populations. Second, CVOTs, by design, recruited subjects with severe cardiovascular comorbidity, but whether outcomes can be generalized to diabetic adults without these comorbidities, or whether heterogeneity of treatment effect varies between ethnicities across levels of disease severity, is not known. Finally, only data reported in the literature were used to make determinations about the generalizability of CVOTs to adult Hispanics/Latinos with diabetes in the USA. Data collected but not reported may provide additional perspective and insight into the applicability of CVOTs to ethnic minorities.

\section{Conclusion}

Diabetes CVOTs have played an important role in evaluating the safety of new diabetes drugs on a global scale; however, they may have limited generalizability to US Hispanic/Latino adults with diabetes. While Hispanic/ Latino representation as a per cent of CVOT subjects 
is comparable with the US adult diabetic population, country of current residence, country of origin, and duration of residence are distinguishing characteristics with potentially important implications for Hispanics/ Latinos that are not available in the CVOT data. As the FDA determines the future role of CVOTs, it should address these limitations by providing enrollment and reporting standards for ethnicity that are aligned with the US diabetes demographic. Providers should consider these limitations when caring for their patients.

\section{Author affiliations}

${ }^{1}$ Department of Internal Medicine, University of Michigan, Ann Arbor, Michigan, USA ${ }^{2}$ Center for Clinical Management Research, Department of Veterans Affairs Ann Arbor Healthcare System, Ann Arbor, Michigan, United States

${ }^{3}$ Institute for Healthcare Policy and Innovation, University of Michigan, Ann Arbor, Michigan, United States

${ }^{4}$ National Clinician Scholars Program, University of Michigan, Ann Arbor, Michigan, United States

${ }^{5}$ Division of Metabolism, Endocrinology and Diabetes, University of Michigan, Ann Arbor, Michigan, United States

${ }^{6}$ Division of General Internal Medicine and Geriatrics, Northwestern University Feinberg School of Medicine, Chicago, Illinois, USA

${ }^{7}$ Departments of Internal Medicine, Pediatrics and Population and Data Sciences, UT Southwestern Medical Center, Dallas, Texas, USA

Acknowledgements The authors acknowledge the editorial assistance of Judith $\mathrm{E}$ Smith, Informationist at the University of Michigan.

Contributors ATTK organized the analysis, drafted and edited the manuscript. $\mathrm{KM}$ assisted in data analysis and editing of the manuscript. MO provided content expertise in developing the study concept and provided edits to drafts. MEB provided content expertise in developing the study concept and provided edits to drafts. JS conceived the study, provided oversight, facilitated development of the study concept, and provided edits to drafts.

Funding No funding was received for this project. Michael Bowen receives research support from a K23 career development award from the National Institute of Diabetes and Digestive and Kidney Diseases (DK104065). Dr. Sussmanis supported by VA CDA 13-021 and VA IIR 15-432. Drs. Kelley and Mizokami-Stout are supported by the National Clinician Scholars Program at the University of Michigan.

Conflicts of interest M0 has received consulting fees from United Healthcare Services and Novo Nordisk that are unrelated this work.

Provenance and peer review Not commissioned; externally peer reviewed.

Data availability statement Data are available upon reasonable request.

Open access This is an open access article distributed in accordance with the Creative Commons Attribution Non Commercial (CC BY-NC 4.0) license, which permits others to distribute, remix, adapt, build upon this work non-commercially, and license their derivative works on different terms, provided the original work is properly cited, appropriate credit is given, any changes made indicated, and the use is non-commercial. See: http://creativecommons.org/licenses/by-nc/4.0/.

\section{REFERENCES}

1. Tucker ME. FDA panel to review 2008 guide on cv trials for diabetes drugs. Secondary FDA panel to review 2008 guide on cv trials for diabetes drugs, 2018. Available: https://www.medscape.com/ viewarticle/903874 [Accessed October 24].

2. Walker RJ, Strom Williams J, Egede LE. Influence of race, ethnicity and social determinants of health on diabetes outcomes. Am J Med Sci 2016;351:366-73.

3. Menke A, Casagrande S, Geiss L, et al. Prevalence of and trends in diabetes among adults in the United States, 1988-2012. JAMA 2015;314:1021-9.

4. Office for Minority Health and Health Equity, Centers for Disease Control and Prevention. CDC vital signs: Hispanic Heatlh. Hispanic Heatlh: Secondary CDC Vital Signs, 2015.

5. Rodríguez JE, Campbell KM. Racial and ethnic disparities in prevalence and care of patients with type 2 diabetes. Clin Diabetes 2017;35:66-70.

6. Mercader JM, Florez JC. The genetic basis of type 2 diabetes in Hispanics and Latin Americans: challenges and opportunities. Front Public Health 2017;5.

7. Ferdinand KC, Nasser SA. Racial/ethnic disparities in prevalence and care of patients with type 2 diabetes mellitus. Curr Med Res Opin 2015;31:913-23.

8. FDA. Food and drug administration safety and Innovation Act, 2012U.S. Food \& Drug Adminstration. Available: https://www.gpo. gov/fdsys/pkg/PLAW-112publ144/pdf/PLAW-112publ144.pdf

9. Bethel MA, Green JB, Milton J, et al. Regional, age and sex differences in baseline characteristics of patients enrolled in the trial evaluating cardiovascular outcomes with sitagliptin (TECOS). Diabetes Obes Metab 2015;17:395-402.

10. Hernandez AF, Green JB, Janmohamed S, et al. Albiglutide and cardiovascular outcomes in patients with type 2 diabetes and cardiovascular disease (harmony Outcomes): a double-blind, randomised placebo-controlled trial. Lancet 2018;392:1519-29.

11. White WB, Cannon CP, Heller SR, et al. Alogliptin after acute coronary syndrome in patients with type 2 diabetes. N Engl J Med 2013;369:1327-35

12. Neal B, Perkovic V, Mahaffey KW, et al. Canagliflozin and cardiovascular and renal events in type 2 diabetes. N Engl J Med 2017;377:644-57.

13. Zinman B, Wanner C, Lachin JM, et al. Empagliflozin, cardiovascular outcomes, and mortality in type 2 diabetes. N Engl J Med 2015;373:2117-28.

14. Holman RR, Bethel MA, Mentz RJ, et al. Effects of once-weekly exenatide on cardiovascular outcomes in type 2 diabetes. $N$ Engl $J$ Med 2017;377:1228-39.

15. Marso SP, Daniels GH, Brown-Frandsen K, et al. Liraglutide and cardiovascular outcomes in type 2 diabetes. $N$ Engl $\mathrm{J}$ Med 2016;375:311-22.

16. Pfeffer MA, Claggett $B$, Diaz R, et al. Lixisenatide in patients with type 2 diabetes and acute coronary syndrome. N Engl J Med 2015;373:2247-57.

17. Scirica BM, Bhatt DL, Braunwald E, et al. Saxagliptin and cardiovascular outcomes in patients with type 2 diabetes mellitus. $N$ Engl J Med 2013;369:1317-26.

18. Marso SP, Bain SC, Consoli A, et al. Semaglutide and cardiovascular outcomes in patients with type 2 diabetes. $N$ Engl $J$ Med 2016;375:1834-44.

19. Green JB, Bethel MA, Armstrong PW, et al. Effect of sitagliptin on cardiovascular outcomes in type 2 diabetes. $N$ Engl J Med 2015;373:232-42.

20. National Center for Health Statistics. National Health and Nutrition Examination Survey. In: Centers for disease control and prevention. ed, 2007-2016.

21. Schneiderman N, Llabre M, Cowie CC, et al. Prevalence of diabetes among Hispanics/Latinos from diverse backgrounds: the Hispanic Community Health Study/Study of Latinos (HCHS/SOL). Diabetes Care 2014;37:2233-9.

22. Kershaw KN, Giacinto RE, Gonzalez F, et al. Relationships of nativity and length of residence in the U.S. with favorable cardiovascular health among Hispanics/Latinos: the Hispanic Community Health Study/Study of Latinos (HCHS/SOL). Prev Med 2016;89:84-9. 